\title{
Morteros y hormigones con cera: ¿ una respuesta a la durabilidad?
}

MaNUel FernandeZ CaNovas, Dr. Ing. de Construcción
I.E.T.c.c.

MARgarita CECILIA Soto, Constructor Civil

I.E.T.c.c.

\section{N T R O D U C C I O N}

El hormigón es, entre los materiales de construcción, si no el más, probablemente uno de los más antiguos. Aún hoy día es posible encontrar vestigios de los hormigones que fabricaron los romanos. Sin embargo, y a pesar de haber sido y de ser ampliamente utilizado, no podemos afirmar que el hormigón haya evolucionado lo que sería de desear. Durante mucho tiempo, el desarrollo y progreso de la técnica mejoró las condiciones de fabricación, de transporte, de puesta en obra, etc.; pero el material en sí sufrió sólo pequeños avances en cuanto a calidad. La calidad del hormigón se ha medido tradicionalmente por su resistencia a la compresión. Pero a menudo, y no obstante gozar de una calidad, el hormigón se ha encontrado frente a problemas de durabilidad.

El hormigón tradicional es el resultado de una mezcla de cemento, grava, arena y agua. Cada uno de estos elementos se va dosificando de forma que se obtenga un material lo más compacto posible. Ahora bien, dentro de esta mezcla existe otro elemento presente: el aire. Sabemos que durante el proceso de hidratación del cemento, parte del agua de amasado se combina con este conglomerante. Otra parte se pierde por simple evaporación. La pérdida del agua de amasado que no se combina con el cemento da origen a pequeños poros de aire. Estos poros de aire conforman una verdadera red de capilares similares al sistema capilar circulatorio de los animales vivos. Pero mientras el sistema capilar de los seres vivos transporta un fluido esencial para mantener esa vida, estos capilares del hormigón sólo pueden transportar materias que pueden causar su muerte, a corto o a largo plazo.

Cuando el hormigón se encuentra en un medio agresivo, por esta red de capilares penetran aquellos elementos nocivos que comenzarán a actuar desde el interior mismo del hormigón, minando poco a poco sus defensas.

Por lo tanto, si se logra encontrar un material que impida el acceso de agentes agresivos, es de suponer que estaremos ante la posibilidad real de mejorar substancialmente la durabilidad del hormigón sin mermar significativamente su resistencia $y$, por ende, su calidad.

Cuando a mediados de los años sesenta se iniciaron los primeros estudios con hormigón polimerizado —utilizando un polímero para rellenar los conductos capilares- se pensó que 
se había llegado a la total solución: mejorar la durabilidad del hormigón y aumentar su resistencia.

Investigaciones anteriores llevadas a cabo en el Instituto Eduardo Torroja (1), han confirmado esta hipótesis. Sin embargo, la utilización de polímeros para sellar estas vías de entrada de los agentes agresivos supone encarecer el costo de fabricación del hormigón, además de aumentar considerablemente la dificultad de dicho proceso, con el agravante de que sólo pueden realizarse en elementos prefabricados.

Ante esta realidad se hace necesario encontrar otro material que actúe satisfactoriamente como sellante de la red capilar del hormigón.

En los años 74-75 se inician, en Estados Unidos, bajo el patrocinio de la Federal Highway Administration (2), una serie de investigaciones conducentes a encontrar un material sellante para prevenir el ataque de los cloruros en carreteras y puentes, ya que las sales utilizadas para evitar la formación de hielo atacan corrosivamente al hormigón. En estos estudios se utilizaron diferentes materiales como sellantes, lográndose un buen comportamiento con una cera ya utilizada en mezclas asfálticas. Se trataba de una cera de naturaleza vegetal cuyo componente más importante es de origen esteárico.

El proceso de fabricación de este hormigón con cera es sencillo. La cera se introduce en la mezcla inicial y se amasa conjuntamente con los demás elementos. Un tratamiento térmico posterior (entre $80^{\circ}$ y $85^{\circ} \mathrm{C}$ ) permite la fusión de los gránulos de cera y el perfecto sellado de los capilares del hormigón. Esta cera que se presenta en forma de polvo contiene un $75 \%$ de parafina, tiene un bajo costo y permite suponer que con ella lograremos un sellado de los capilares y, por tanto, un mejor comportamiento del hormigón en medios agresivos y una mayor durabilidad.

En los trabajos de investigación que hemos realizado no se tenía como meta encontrar un material sellante capaz de reemplazar a los hormigones impregnados de polímeros por los hormigones cera, debido, entre otras cosas, a que con la polimerización se busca también fuertes incrementos de resistencias mecánicas. Sólo se ha pretendido encontrar un sustituto de menor costo, de fabricación y tratamiento posterior más sencillo que permitan utilizar este hormigón en forma más masiva y en extensas zonas de suelos agresivos como las existentes en España y en el norte de Chile. En ellas los sulfatos y cloruros existentes atacan despiadadamente a los hormigones tradicionales. Muchos elementos prefabricados como postes, tuberías, canalizaciones, etc., deben ser frecuentemente reemplazados por otros materiales que no sufran ataque químico por parte de estos suelos. El hormigón polimerizado es aún un material de élite para estos usos. Creemos y confiamos en que el hormigón-cera puede ser el material ideal para estas zonas.

Los resultados que hemos obtenido en esta primera parte, son altamente alentadores. Tanto los morteros-cera como los hormigones-cera muestran mejoras apreciables en cuanto a durabilidad sin bajas significativas en sus resistencias.

Pero no adelantemos conclusiones. Echemos primero un vistazo al proceso de fabricación, a los ensayos realizados y finalmente extraigamos las conclusiones oportunas. Creemos que queda aún mucho por ver y estudiar acerca de la durabilidad del hormigón y cómo mejorarla. Los hormigones-cera son un paso más adelante.

\section{MATERIALES}

En el estudio realizado hemos utilizado tanto para la fabricación de los morteros como de los hormigones, cemento portland con adiciones activas (PA-350). 
Los áridos fueron de tipo silíceo y de canto rodado, procedente de río, los cuáles mantenían una granulometría constante durante toda la investigación. Para el caso del hormigón se ha empleado árido de $20 \mathrm{~mm}$ de tamaño máximo, debido a las dimensiones de las probetas empleadas.

En cuanto al material cuyo comportamiento con el hormigón estaba en estudio, se ha empleado una cera montana con $75 \%$ de parafina. Esta es una cera vegetal, fosilizada, de características similares a las ceras carnaubas, la cual es obtenida a partir del lignito, mediante técnicas de extracción con disolventes orgánicos. Su composición es de carácter predominantemente alifático, formada por ésteres y cantidades no significativas de cetonas y ácidos carboxílicos de larga cadena.

Características importantes de esta cera con relación a nuestro trabajo eran:

- Punto de fusión

- Indice de acidez

- Indice de saponificación 83 a 102

La granulometría de las esferas de cera era la siguiente:

\begin{tabular}{c} 
Tamiz (mm) \\
\hline 0,59 \\
0,29 \\
0,14 \\
0,07
\end{tabular}

\begin{tabular}{c} 
Retenido (\%) \\
\hline 0,0 \\
70,9 \\
24,5 \\
3,8 \\
0,8
\end{tabular}

\section{PROCESO DE FABRICACION}

\subsection{Morteros}

En una primera etapa se procedió a estudiar la influencia de la cera sobre los morteros. Los porcentajes de cera seleccionados para realizar este estudio fueron del 1, 2, 2,5 y $3 \%$ con respecto al peso del mortero.

El número de series fabricadas está detallado en el "Cuadro de Plan de Ensayos para Morteros" que se incluye en el Apartado 3.

Las dosificaciones empleadas fueron las que se indican en el Cuadro 2.1.

C UADRO 2.1.

Dosificación de morteros

\begin{tabular}{|c|c|c|c|cc|}
\hline Designación & $\begin{array}{c}\text { Arena } \\
(\mathrm{g})\end{array}$ & $\begin{array}{c}\text { Cemento } \\
(\mathrm{g})\end{array}$ & $\begin{array}{c}\text { Agua } \\
(\mathrm{g})\end{array}$ & \multicolumn{2}{|c|}{ Cera } \\
\hline M-A & 1.500 & 500 & 250 & \multicolumn{2}{|c|}{0} \\
M-B & 1.500 & 500 & 250 & 22,50 & 1 \\
M-C & 1.500 & 500 & 250 & 45 & 2 \\
M-D & 1.500 & 500 & 250 & 56,25 & 2,5 \\
M-E & 1.500 & 500 & 250 & 67,50 & 3 \\
\hline
\end{tabular}


Con cada mortero se fabricaron 10 series de tres probetas prismáticas de $4 \times 4 \times 16 \mathrm{~cm}$. Tres series se introdujeron en baños agresivos, dos series se sometieron a tratamiento térmico a los 14 días y se rompieron a compresión y flexotracción a los 28 y 90 días, dos series se rompieron a compresión y flexotracción sin tratamiento térmico a los 28 y 90 días, una serie se sometió a ensayos de absorción, otra a ensayo de capilaridad y finalmente una serie a ensayo de heladicidad.

Las probetas se desmoldaron a las 24 horas, manteniéndose en cámara de curado durante 13 días, $95 \%$ de humẹdad relativa y a $20^{\circ} \pm 1{ }^{\circ} \mathrm{C}$ de temperatura.

\section{Tratamiento térmico}

Ocho de las series fabricadas se retiraron de la cámara de curado a los 13 días para ser sometidas 24 horas después al tratamiento térmico necesario para lograr la fusión de la cera. Este tratamiento consistió en calentar durante 3 horas a $40^{\circ} \mathrm{C}$ en estufa. A continuación y por espacio de 3 se mantuvo la temperatura a $85^{\circ} \mathrm{C}$ para posteriormente enfriar a $40^{\circ} \mathrm{C}$ por otras 3 horas. Luego se retiraron las probetas de la estufa, permaneciendo en ambiente de laboratorio hasta cumplir el tiempo necesario para ser sometidas al ensayo que correspondiera.

\subsection{Hormigones}

Para el estudio sobre hormigón se seleccionaron tres porcentajes de cera: 2, 2,5 y $3 \%$ de cera con respecto al peso del hormigón. El número de probetas fabricadas están detalladas en el Cuadro de Plan de Ensayo del Hormigón que se incluye en el Apartado 3.

Las dosificaciones empleadas fueron las que se indican en el Cuadro 2.2.

\section{UADRO 2.2 .}

Dosificación de hormigones

\begin{tabular}{|c|c|c|c|c|cc|}
\hline Designación & $\begin{array}{c}\text { Arena } \\
(\mathbf{k g})\end{array}$ & $\begin{array}{c}\text { Pińnoncillo } \\
(\mathbf{k g})\end{array}$ & $\begin{array}{c}\text { Cemento } \\
(\mathbf{k g})\end{array}$ & $\begin{array}{c}\text { Agua } \\
(\mathbf{k g})\end{array}$ & (kg) & Cera \\
\hline H-A & 13,3 & 20,0 & 7,0 & 3,65 & - & 0 \\
H-B & 13,3 & 20,0 & 7,0 & 3,65 & 0,879 & 2 \\
H-C & 13,3 & 20,0 & 7,0 & 3,65 & 1,098 & 2,5 \\
H-D & 13,3 & 20,0 & 7,0 & 3,65 & 1,318 & 3 \\
\hline
\end{tabular}

Los hormigones se amasaron en hormigonera basculante, mezclándose primero en seco, el cemento, los áridos y la cera. Luego se introdujeron con la mitad del agua de amasado en la hormigonera, agregándose posteriormente el resto del agua. En todas las amasadas se realizó la prueba de asentamiento del cono de Abrams, siendo su consistencia seco-plástica.

\section{Tratamiento térmico}

A los 14 días se sometieron a tratamiento térmico las probetas de hormigón-cera y de hormigón normal correspondientes al programa de ensayos. El tratamiento térmico a que fueron sometidas se realizó en forma idéntica al ya indicado para los morteros. Con posterio- 
ridad al tratamiento, las probetas tratadas quedaron en ambiente de laboratorio, manteniéndose las no tratadas en la cámara de curado hasta la edad en que serían sometidas al ensayo correspondiente.

\section{PLAN DE ENSAYOS Y RESULTADOS OBTENIDOS}

Cuando iniciamos este trabajo sobre los morteros-cera y los hormigones-cera, y su comportamiento en medios agresivos, debimos fijarnos algunos índices o valores que nos permitieran evaluar dicho comportamiento.

Sabido es que los ensayos de durabilidad frente a agentes agresivos, que se realizan sumergiendo las probetas de hormigón o mortero en estanques saturados con el agresivo son, básidamente, ensayos de larga duración. Por este motivo, además de planificar la realización de ensayos de este tipo, decidimos realizar paralelamente otros ensayos que nos dieran una pauta acerca del posible comportamiento del hormigón-cera y del morterocera en ambientes agresivos.

Por ello sometimos tanto a los morteros como a los hormigones a ensayos que nos permitieran evaluar su calidad relativa, como son los ensayos de rotura, o su resistencia a la penetración mediante ensayos de absorción, capilaridad y heladicidad.

\subsection{Morteros}

El Plan de Ensayo a que fueron sometidos los cinco tipos de morteros confeccionados se indica en el Cuadro 3.1.1. En el mismo se recogen el número de series de 3 probetas cada una que se empleó en cada ensayo.

CUADRO 3.1.1.

Plan de ensayos para morteros

\begin{tabular}{|c|c|c|c|c|c|c|c|}
\hline \multirow{2}{*}{ Mortero } & \multirow{2}{*}{$\begin{array}{l}\text { Ensayo de } \\
\text { absorción }\end{array}$} & \multirow{2}{*}{\begin{tabular}{|} 
Ensayo de \\
capilaridad
\end{tabular}} & \multirow{2}{*}{$\begin{array}{l}\text { Ensayo de } \\
\text { heladicidad }\end{array}$} & \multirow{2}{*}{$\begin{array}{cl}\text { Resistencia a } & \begin{array}{l}\text { Resistencia a } \\
\text { compresión }\end{array} \\
\text { flexotracción }\end{array}$} & \multicolumn{3}{|c|}{ Durabllidad } \\
\hline & & & & & $\mathrm{NaSO}_{4}$ & $\mathrm{MgSO}_{4}$ & HCl \\
\hline Normal & - & - & - & 2 series rotas a 28 y 90 días & - & - & - \\
\hline Normal tt. & 1 & 1 & 1 & 2 series rotas a 28 y 90 días & 1 & 1 & 1 \\
\hline $1 \%$ cera ntt. & - & - & - & 2 series rotas a 28 y 90 dias & - & - & - \\
\hline $1 \%$ cera tt. & 1 & 1 & 1 & 2 series rotas a 28 y 90 días & 1 & 1 & 1 \\
\hline $2 \%$ cera ntt. & - & - & - & 2 series rotas a 28 y 90 días & - & - & - \\
\hline $2 \%$ cera tt. & 1 & 1 & 1 & 2 series rotas a 28 y 90 días & 1 & 1 & 1 \\
\hline 2,5 cera $n t t$. & - & - & - & 2 series rotas a 28 y 90 dias & - & - & - \\
\hline 2,5 cera tt. & 1 & 1 & 1 & 2 series rotas a 28 y 90 días & 1 & 1 & 1 \\
\hline $3 \%$ cera ntt. & - & - & - & $\approx$ series rotas a 28 y 90 dias & - & - & - \\
\hline $3 \%$ cera tt. & 1 & 1 & 1 & 2 series rotas a 28 y 90 dias & 1 & 1 & 1 \\
\hline
\end{tabular}

NOTA :

tt. = tratada térmicamente.

ntt. = no tratada térmicamente. 


\section{a) Ensayo de resistencia a compresión y flexotracción}

Tal como se indica en el Cuadro 3.1.1., se rompieron probetas a 28 y 90 días, con y sin tratamiento térmico, con los cuatro porcentajes de cera seleccionados. Los resultados medios obtenidos se indican en el Cuadro 3.1.2., no habiéndose obtenido grandes dispersiones en los mismos.

C U A D R O 3.1.2.

Resistencias medias a compresión y flexotracción en morteros

\begin{tabular}{|c|c|c|c|c|}
\hline \multirow{2}{*}{ Motero } & \multicolumn{2}{|c|}{ Compresión $\left(\mathrm{kp} / \mathrm{cm}^{2}\right)$} & \multicolumn{2}{|c|}{ Flexotracción $\left(\mathrm{kp} / \mathrm{cm}^{2}\right)$} \\
\hline & 28 días & 90 días & 28 dias & 90 dias \\
\hline Normal & 459,1 & 492,1 & 77,7 & 79,6 \\
\hline Normal tt. & 413,0 & 456,3 & & \\
\hline $1 \%$ cera ntt. & 432,3 & 465,2 & 65,8 & 83,2 \\
\hline $1 \%$ cera tt. & 387,0 & 414,7 & 78,0 & 91,3 \\
\hline $2 \%$ cera ntt. & 418,1 & 451,5 & 50,2 & 71,5 \\
\hline $2 \%$ cera tt. & 359,0 & 380,8 & 74,4 & 91,9 \\
\hline 2,5 \% cera ntt. & 402,3 & 422,9 & 65,9 & 84,5 \\
\hline $2,5 \%$ cera tt. & 353,7 & 372,3 & 77,7 & 90,0 \\
\hline $3 \%$ cera ntt. & 373,1 & 394,7 & 59,5 & 77,3 \\
\hline $3 \%$ cera tt. & 317,2 & 333,1 & 61,3 & 86,6 \\
\hline
\end{tabular}

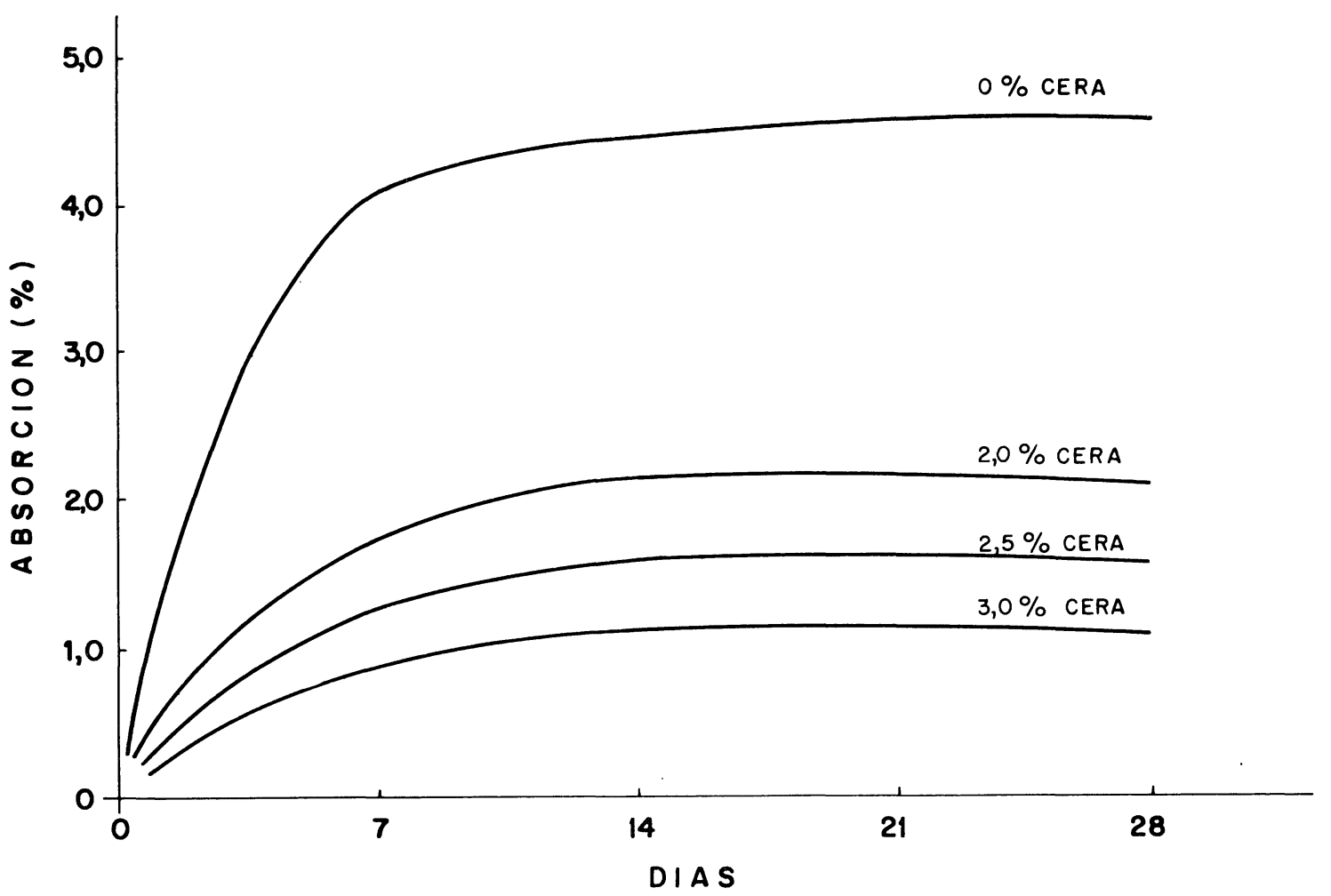

Gráfico 3.1.1. 


\section{b) Ensayo de absorción}

A este ensayo se sometieron series tratadas térmicamente tal como se desprende del Plan de Ensayos, con objeto de que las probetas tuvieran inicialmente un peso constante. Las probetas se pesaron y se sumergieron en un depósito con agua. Se sometieron a distintas pesadas hasta que la absorción fue constante. En el Gráfico 3.1.1. se han dibujado las curvas de absorción, teniendo como origen el día en que inciamos el ensayo, en abscisas la edad y en ordenadas el \% de absorción.

\section{c) Ensayo de capilaridad}

Al iniciar el ensayo fueron pesadas las probetas; seguidamente se colocaron en un recipiente de forma que sólo un tercio de la probeta permaneciera sumergida, es decir, la altura del agua se mantuvo constante, durante el período de duración del ensayo, a $5 \mathrm{~cm}$. Los resultados obtenidos se han representado en el Gráfico 3.1.2. con una curva de capilaridad para cada tipo de mortero.

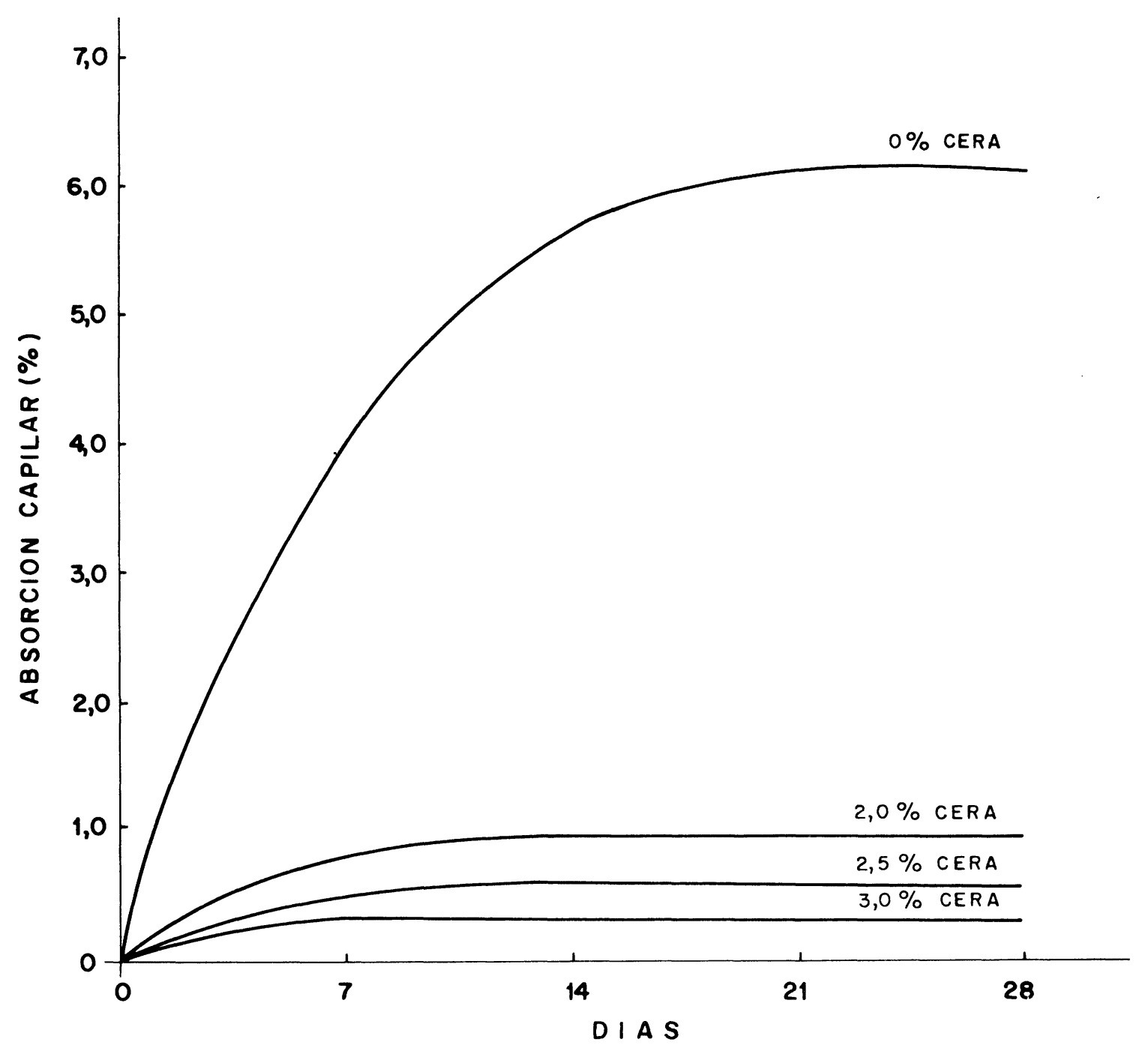

Grafico 3.1.2. 


\section{d) Ensayo de heladicidad}

Las probetas de mortero han sido sometidas a 135 ciclos de hielo/deshielo, observándose fallos muy apreciables en las probetas de mortero normal a partir del ciclo 83, tal como se aprecia en la figura 1. En la figura, los morteros sin cera habían experimentado 85 ciclos mientras que los otros morteros habían experimentado 135 ciclos.

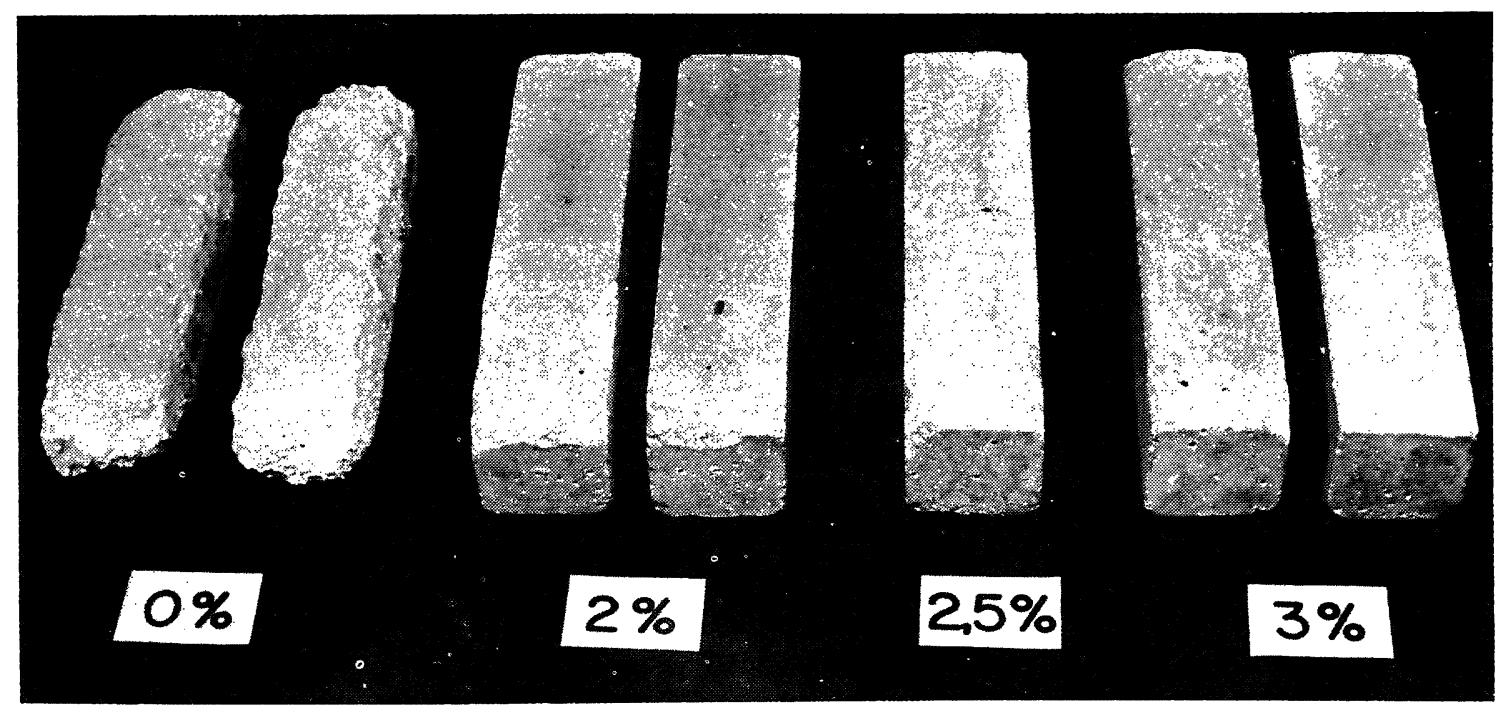

Fig. 1

El porcentaje de pérdida de peso a este número de ciclos fue:

- Mortero $0 \%$ de cera $\ldots \ldots \ldots \ldots \ldots \ldots \ldots \ldots \ldots \ldots \ldots$

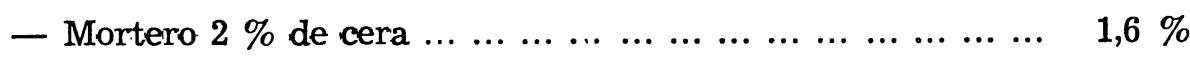

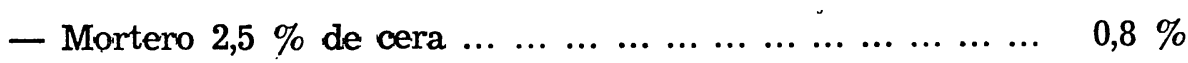

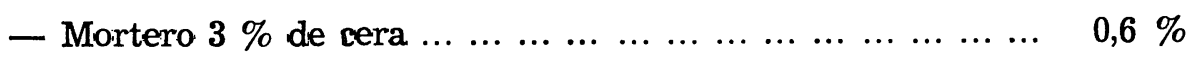

e) Ensayo de durabilidad

Anteriormente hemos indicado que los ensayos de durabilidad por inmersión de probetas, en medios agresivos, son ensayos de larga duración. Para paliar en parte esta dificultad y obtener unos primeros resultados, sometimos las probetas a la acción de ataque acelerado con reactivos concentrados.

Los agresivos que seleccionamos fueron tres: sulfato magnésico $\left(\mathrm{MgSO}_{4}\right)$, sulfato sódico $\left(\mathrm{Na}_{2} \mathrm{SO}_{4}\right)$ y ácido clorhídrico $(\mathrm{HCl})$. Las concentraciones fueron del $10 \%, 10 \%$ y $15 \%$ respectivamente.

A los 120 días de iniciado este ensayo extrajimos una probeta de cada tipo de mortero y de cada baño agresivo. Por medio de una máquina de corte refrigerada con aire comprimido, se tallaron pequeños testigos a distintas profundidades, los que sometimos a análisis químico para determinar el porcentaje de agresivo presente a dichas profundidades.

Estos resultados se encuentran reseñados en en el Cuadro 3.1.3. y se obtuvieron después de una permanencia de 4 meses en los baños agresivos. 
C UADRO 3.1.3.a

Determinación de cloruros en morteros

\begin{tabular}{|c|c|c|c|c|}
\hline Probetas & Normal & $2 \%$ & $2,5 \%$ & $3 \%$ \\
\hline Exterior & $0,54 \%$ & $0,25 \%$ & $0,19 \%$ & $0,17 \%$ \\
\hline Interior & $0,49 \%$ & $0,17 \%$ & $0,13 \%$ & $0,11 \%$ \\
\hline
\end{tabular}

C UADRO 3.1.3.b

Determinación de $\mathrm{Na}_{2} \mathrm{SO}_{4}$ en morteros

\begin{tabular}{|c|c|c|c|c|}
\hline Probetas & Normal & $2 \%$ & $2,5 \%$ & $3 \%$ \\
\hline Exterior & $1,7 \%$ & $1,5 \%$ & $1,5 \%$ & $1,3 \%$ \\
\hline Interior & $1,4 \%$ & $1,3 \%$ & $1,3 \%$ & $1,0 \%$ \\
\hline
\end{tabular}

C U A D R O 3.1.3.c.

Determinación de $\mathrm{MgSO}_{4}$ en morteros

\begin{tabular}{|c|c|c|c|c|}
\hline Probetas & Normal & $2 \%$ & $2,5 \%$ & $3 \%$ \\
\hline Exterior & $1,6 \%$ & $1,4 \%$ & $1,4 \%$ & $1,1 \%$ \\
\hline Interior & $1,5 \%$ & $1,3 \%$ & $1,3 \%$ & $1,0 \%$ \\
\hline
\end{tabular}

A los 300 días de tener sumergidas las probetas en los líquidos agresivos, el estado que mostraban éstas queda recogido en las fíguras 2,3 y 4 .

Se observa un mayor ataque en la disolución de $\mathrm{MgSO}_{4}$, seguido del $\mathrm{Na}_{2} \mathrm{SO}_{4}$ y $\mathrm{HCl}$.

El ataque es muy acentuado en los morteros sin cera, es decir, tradicionales, mientras que a esta edad los morteros con cera no muestran alteración visible en los baños de sulfatos y sí un ligero ataque en el baño de ácido clorhídrico. 


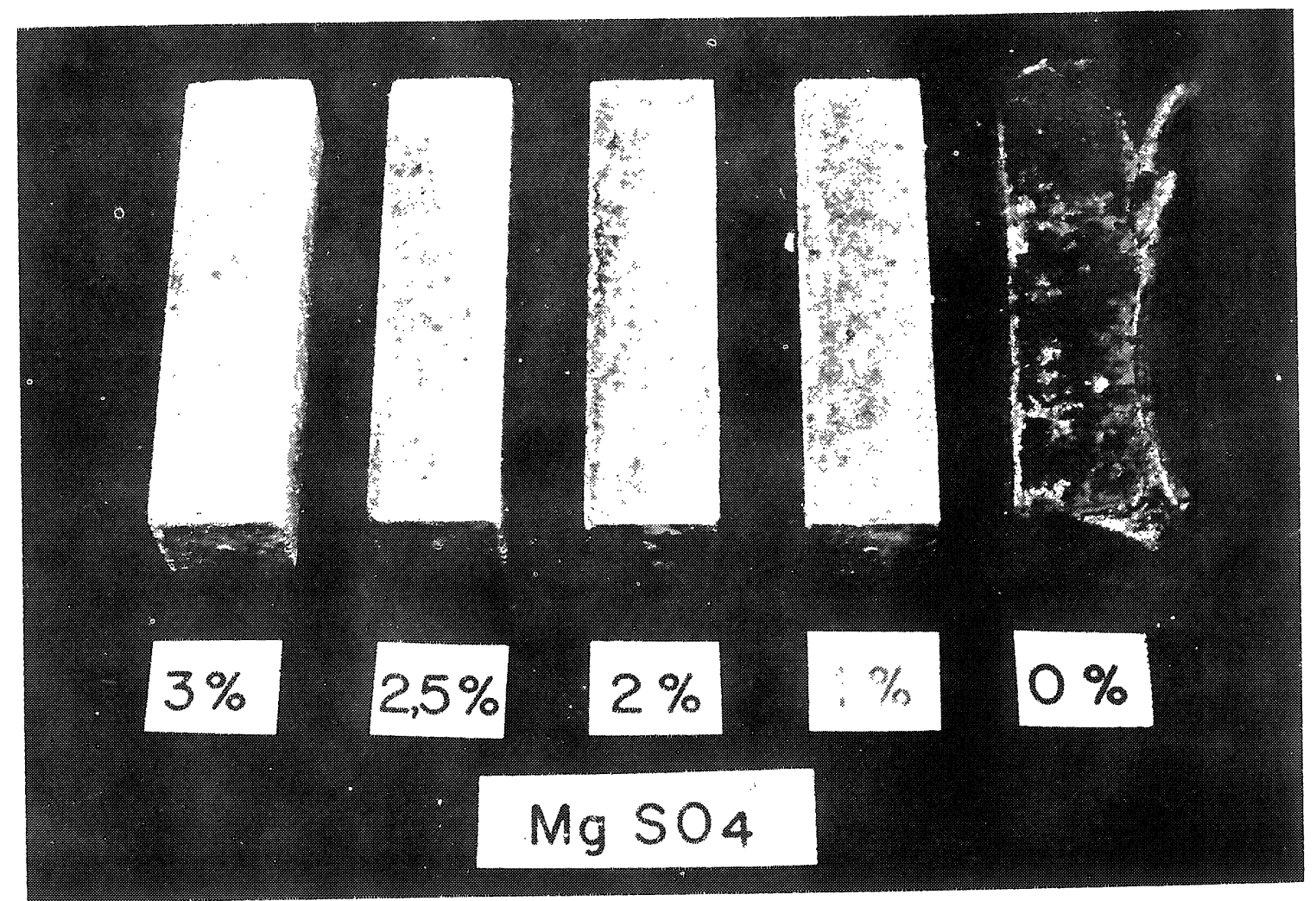

FIg. 2

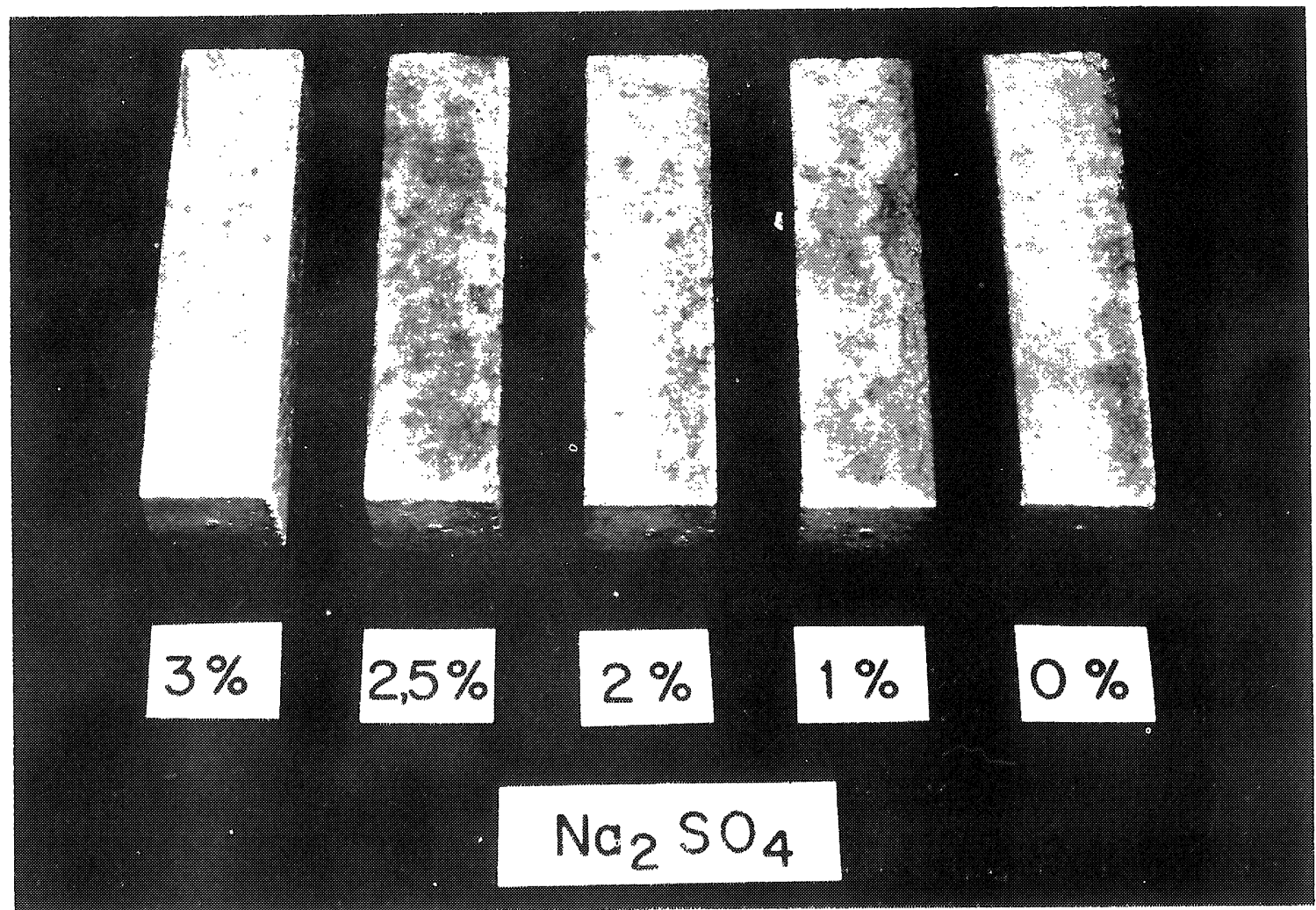

ํㅣㄹ. 3 


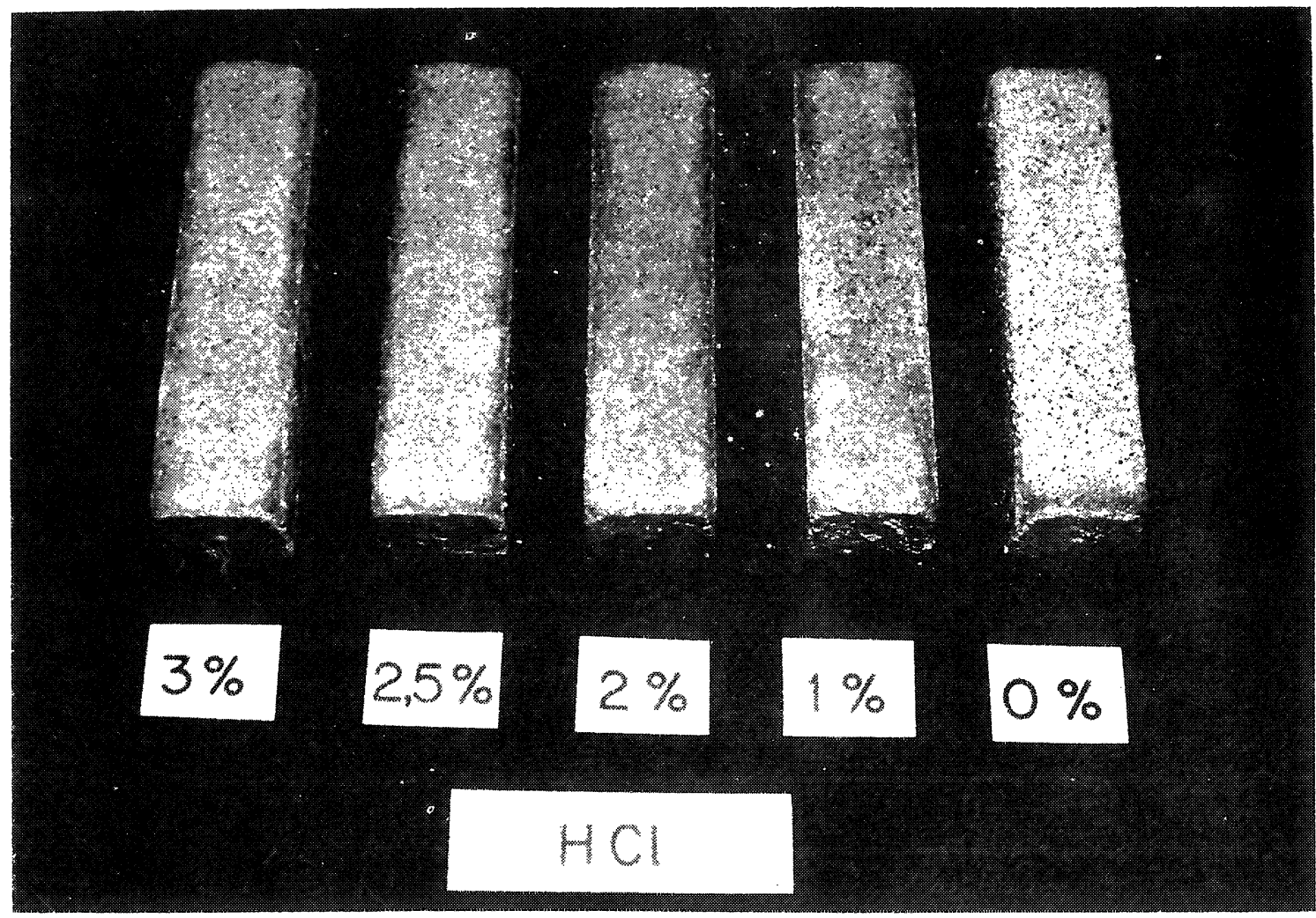

\subsection{Hormigones}

Fig. 4

El Plan de Ensayos a que fueron sometidos los distintos tipos de hormigón confeccionado se indica en el Cuadro 3.2.1. En el mismo se recoge el número de probetas cilíndricas de $7,5 \varnothing \times 15 \mathrm{~cm}$, que se emplearon en cada ensayo.

CUADRO 3.2.1.

Plan de ensayos para hormigones

\begin{tabular}{|c|c|c|c|c|c|c|}
\hline \multirow{2}{*}{ Wormigones } & \multirow{2}{*}{$\begin{array}{l}\text { Resistencia a } \\
\text { compresión }\end{array}$} & \multirow{2}{*}{$\begin{array}{l}\text { Ensayo de } \\
\text { absorción }\end{array}$} & \multirow{2}{*}{$\begin{array}{l}\text { Ensayo de } \\
\text { rapilaridad }\end{array}$} & \multicolumn{3}{|c|}{ Durabilidad } \\
\hline & & & & $\mathrm{Na}_{2} \mathrm{NO}_{4}$ & $\mathrm{MgSO}_{4}$ & IIICI \\
\hline Normal & 9 & - & - & - & - & - \\
\hline Normal tt. & 6 & 3 & 3 & 3 & 3 & 3 \\
\hline $2 \%$ cera ntt. & 6 & - & - & - & - & - \\
\hline $2 \%$ cera tt. & 6 & - & - & - & - & - \\
\hline $2,5 \%$ cera ntt. & 9 & - & - & - & - & - \\
\hline $2,5 \%$ cera tt. & 6 & 3 & 3 & 3 & 3 & 3 \\
\hline $3 \%$ cera ntt. & 6 & - & - & $-\cdots$ & - & $\ldots$ \\
\hline $3 \%$ cera $t t$. & 6 & - & - & 3 & $a$ & 3 \\
\hline
\end{tabular}

NTOR:

A. -. Tumada tirmicamente.

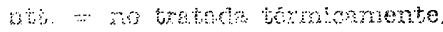




\section{a) Ensayo de resistencia a compresión}

Los resultados de la rotura a compresión de los hormigones-cera y hormigón normal se indican en el Cuadro 3.2.2. y las curvas resistencia vs. edad, se encuentran representadas gráficamente para cada tipo de hormigón en el gráfico 3.2.1.

CUADRO 3.2.2.

Resistencias medias a compresión en hormigones

\begin{tabular}{|c|c|c|c|}
\hline \multirow{2}{*}{ Hormigones } & \multicolumn{3}{|c|}{ Compresión $\left(\mathbf{k p} / \mathrm{cm}^{2}\right)$} \\
\cline { 2 - 4 } & 7 dias & 28 dias & 90 dias \\
\hline Normal & 301 & 362 & 403,7 \\
\hline Normal tt. & & 312 & 376 \\
\hline $2 \%$ cera & 223,4 & 308,2 & 364,3 \\
\hline $2 \%$ cera tt. & & 274,8 & 321,2 \\
\hline $2,5 \%$ cera & 206,5 & 262,3 & 318,4 \\
\hline $2,5 \%$ cera tt. & & 246,7 & 270,1 \\
\hline $3 \%$ cera & 199,4 & 257,2 & 282,3 \\
\hline $3 \%$ cera tt. & & 242,8 & 247,5 \\
\hline
\end{tabular}

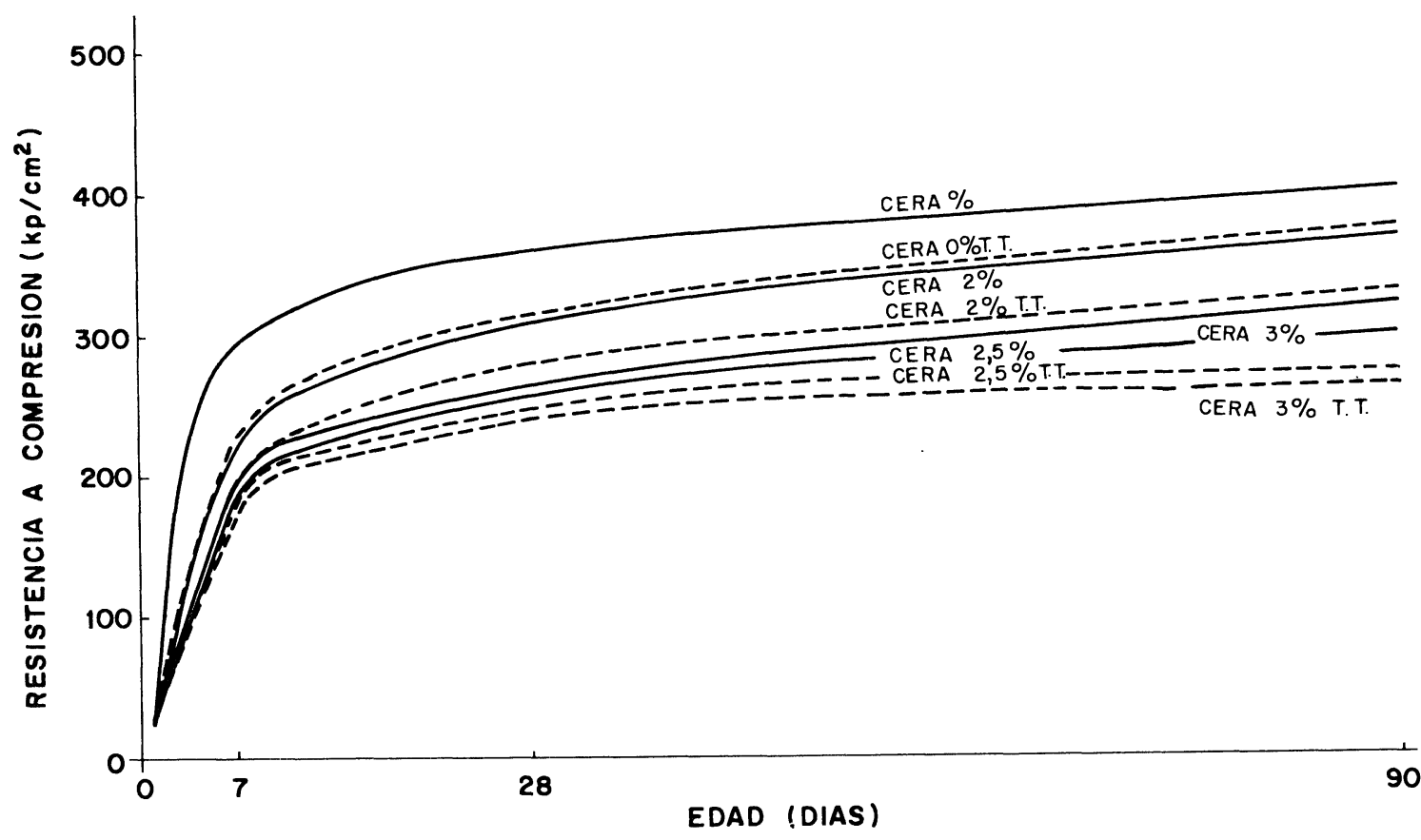

Gráfico 3.2.1. 


\section{b) Ensayo de absorción}

De acuerdo con el Plan de Ensayo se sometieron a ensayo de absorción una serie de hormigón normal y una serie de hormigón-cera al 2,5 \%, ambas tratadas térmicamente a los 14 días y que tenían un peso constante en el momento de ser sumergidas en agua. La curva de absorción se obtuvo pesando las probetas a distintas edades hasta obtener un peso constante y, por ende, un porcentaje de absorción constante. Las curvas obtenidas para cada hormigón están representadas en el Gráfico 3.2.2.

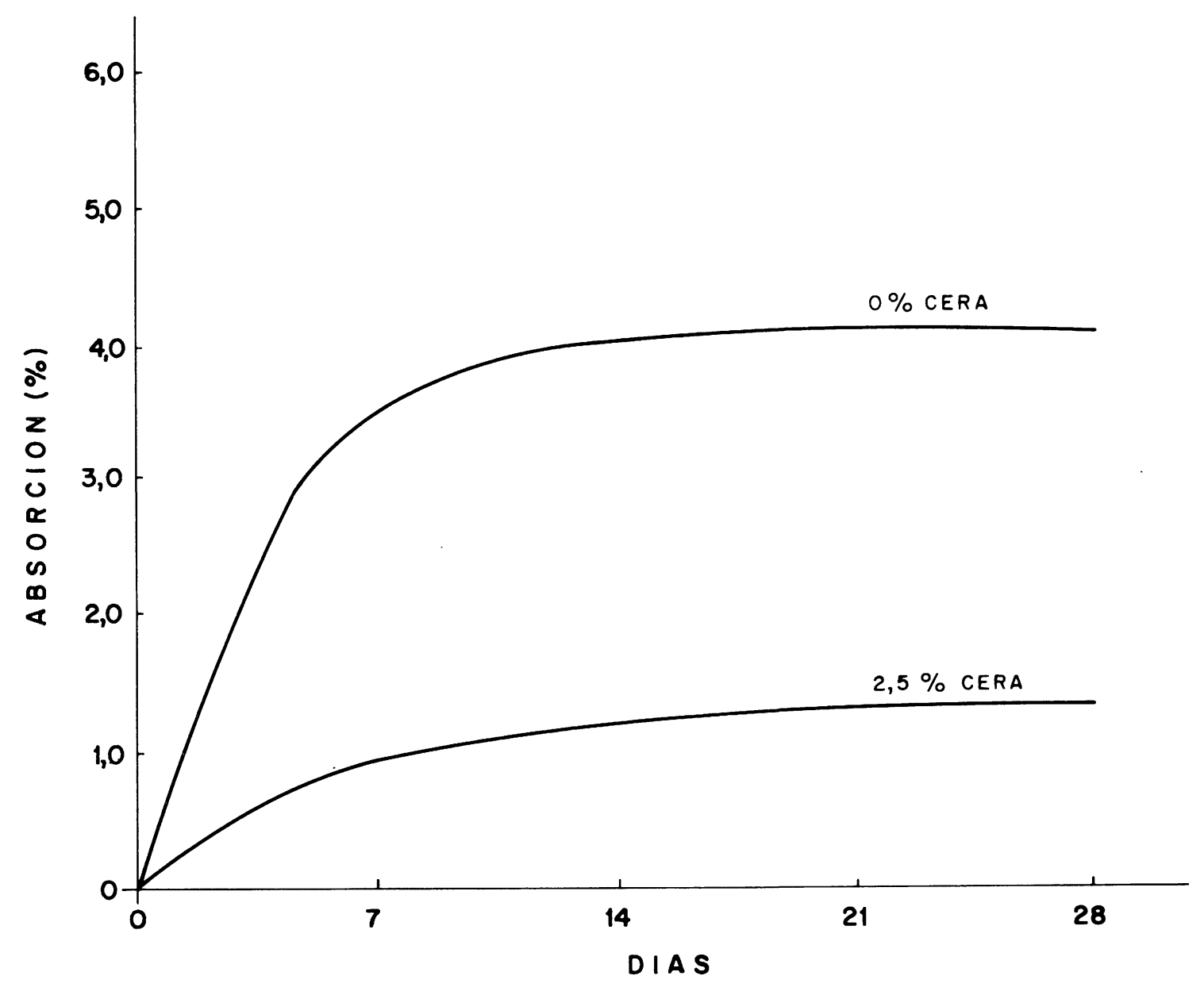

Gráfico 3.2.2.

c) Ensayo de capilaridad

En este ensayo también se emplearon dos tipos de hormigones: una serie de hormigón normal y una serie de hormigón-cera al 2,5 \%, ambas tratadas térmicamente a los 14 días. Estas probetas se pesaron y se sumergieron en agua hasta quedar con un tercio de su altura en contacto directo con el agua. Se realizaron pesadas durante un período de tiempo hasta obtener valores constantes de los pesos. Las curvas de capilaridad obtenidas se han representado en el Gráfico 3.2.3.

d) Ensayos de durabilidad

Al igual que en los morteros, se seleccionaron tres baños agresivos con concentraciones fuertes. Estos agresivos fueron: sulfato sódico $\left(\mathrm{Na}_{2} \mathrm{SO}_{4}\right)$, sulfato magnésico $\left(\mathrm{MgSO}_{4}\right)$ y ácido clorhídrico $(\mathrm{HCl})$, siendo sus concentraciones del $10 \%, 10 \%$ y $15 \%$ respectivamente. 


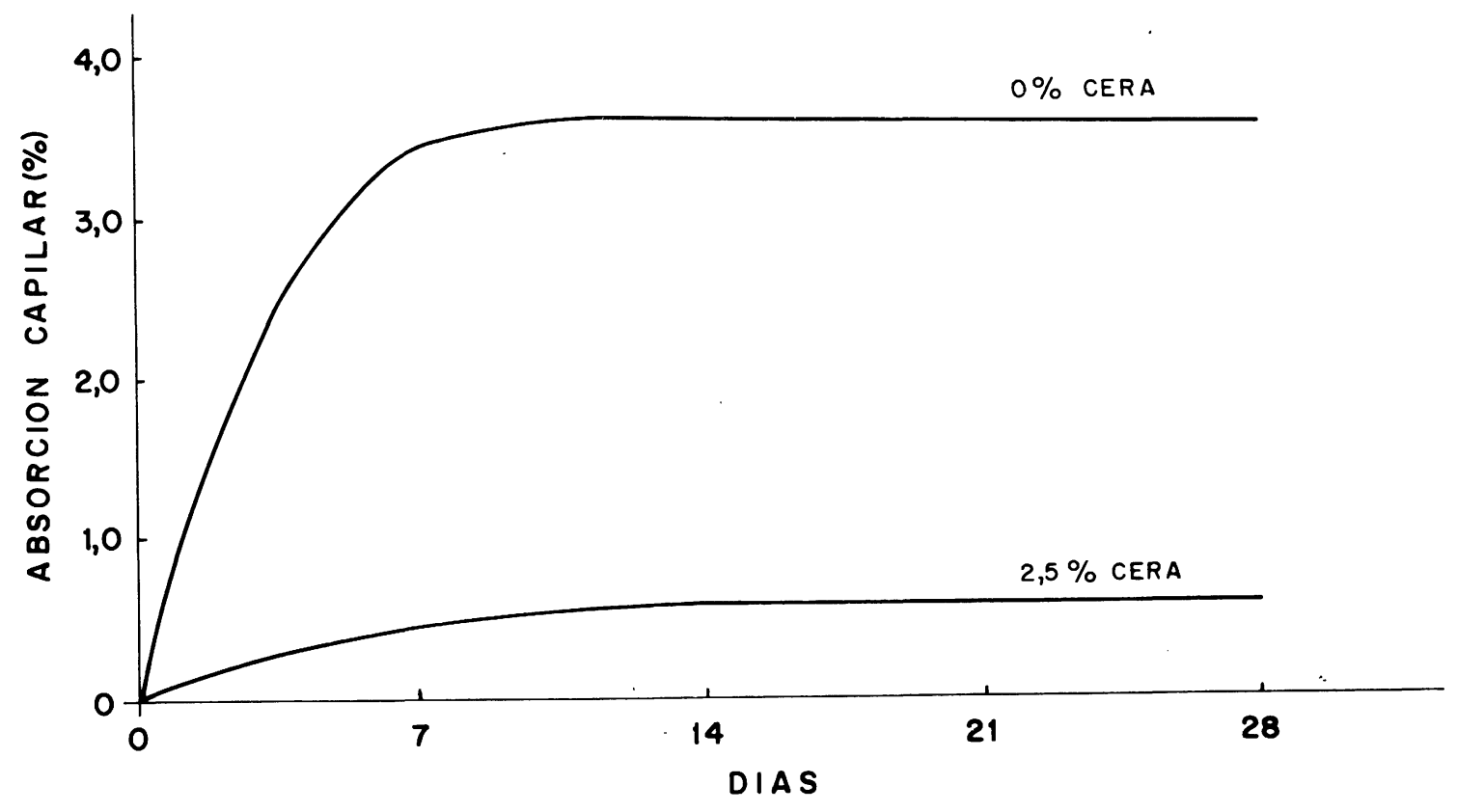

Gráfico 3.2.3.

Los resultados de los ensayos muestran un claro ataque preferencial hacia los hormigones que no tenían cera, siendo menor el ataque cuanto mayor es el contenido de cera.

\section{CONCLUSIONES}

De acuendo con los resultados obtenidos, podemos extraer algunas conclusiones generales y válidas tanto para morteros como para hormigones.

- Como era de esperar, las resistencias mecánicas disminuyen a medida que aumenta la presencia de cera, pero esta disminución no es significativa y es debida, por lo menos en un $10 \%$ aproximadamente, al tratamiento térmico a que fueron sometidas las probetas.

- La cera no altera el resto de las propiedades del material, ya sea mortero u hormigón.

- En nuestra introducción decíamos que la penetración de agentes agresivos se producía por los capilares del material. Los resultados de los ensayos de absorción de agua y de capilaridad nos muestran unos morteros y unos hormigones sellados a tal punto que la capacidad de absorción disminuye a una cuarta parte en morteros y a una sexta en hormigones. La capilaridad disminuye a una quinta parte en morteros y a una novena en hormigones. Luego, en esta misma proporción estamos disminuyendo la posibilidad de penetración de agentes agresivos.

- Los mejores resultados a la vista de todos los ensayos realizados se han obtenido con una adición de un 2,5\% de cera, tanto en hormigones como en morteros, ya que a partir de este porcentaje las bajas de resistencia mecánica comienzan a ser más significativas.

- El porcentaje de agresivo presente en las muestras interiores analizadas, es decir, a una profundidad de $13 \mathrm{~mm}$, demuestra que sólo una cuarta parte del agresivo que pe- 
netra en una probeta de mortero normal llega a esa profundidad en probetas de mortero con un 2,5\% de cera y en el caso del ácido clorhídrico en el cual se utilizó la concentración más fuerte.

- Esta es una primera parte de las investigaciones que estamos realizando sobre los morteros y hormigones con cera. Quedan aún algunas incógnitas por desvelar. En gran medida son aquellas que dicen relación con el comportamiento a más largo plazo de este material en medios agresivos. Una primera tentativa de ensayo electroquímico para determinar la corrosión del acero en el interior de una probeta, realizada con un porcentaje de cera muy bajo, nos indica que también en este aspecto queda un gran campo de investigación por desarrollar.

\section{B I B L I O G R A F I A}

(1) F. Arredondo; M. F. Canovas; J. Fontan; E. L. Madruga: "Mejora del hormigón mediante su impregnación con polímeros vinílicos", monografía núm. 345 del I.E.T. Octubre 1977.

(2) G. H. JENkINS: "Internally sealed concrete”. Federal Highway Administration. Enero 1975.

(3) K. C. Clear; S. W. FORSteR: "Internally sealed concrete: material, characterization and heat treating studies". Federal Highway Administration. Marzo 1977. 